\title{
Corticoterapia na sepse: uma revisão integrativa de literatura
}

\author{
Corticotherapy in sepsis: an integrative literature review \\ Corticoterapia en la sepsis: revisión integradora de la literatura
}

Laila Caroline Silva Sousa

ORCID: https://orcid.org/0000-0002-9632-8208

Centro Universitário de Patos de Minas, Brasil

E-mail: lailacarolline23@ hotmail.com

Bárbara Queiroz de Figueiredo

ORCID: https://orcid.org/0000-0003-1630-4597

Centro Universitário de Patos de Minas, Brasil

E-mail: barbarafigueiredo@unipam.edu.br

Dalbert Samuel Dutra

ORCID: https://orcid.org/0000-0001-8417-1138

Centro Universitário de Patos de Minas, Brasil E-mail: dalbertdutra@gmail.com

Elizabethe Damiani

ORCID: https://orcid.org/0000-0003-0306-1389

Centro Universitário de Patos de Minas, Brasil elizabethe.damiani@hotmail.com

Luis Ferreira Neto

ORCID: https://orcid.org/0000-0003-4397-2283 Universidade de Uberaba, Brasil

E-mail: trabalho.luis99@gmail.com

Lunalva Gabrielli Veras Sousa

ORCID: https://orcid.org/0000-0001-5181-5371

Centro Universitário Uninovafapi, Brasil E-mail: veraslunalva@gmail.com

Rúbia Carla Oliveira

ORCID: https://orcid.org/0000-0002-7162-3497

Centro Universitário de Patos de Minas, Brasil E-mail: rubiacoliveira@unipam.edu.br

\begin{abstract}
Resumo
Introdução: a sepse consiste em uma doença inflamatória sistêmica associada a uma infecção com manifestações graves que ocorre por todo o organismo. No entanto, essa infecção não precisa estar necessariamente em todos os locais do organismo, pode estar restrita em apenas um local, mas causar uma resposta inflamatória por todo o corpo para tentar controlar a causa da infecção Objetivo: demonstrar as atuais evidências sobre o uso de corticosteroides no tratamento da sepse em paciente hospitalizados nas unidades de terapia intensiva (UTI's). Metodologia: trata-se de uma pesquisa descritiva do tipo revisão integrativa da literatura. Foi realizado o cruzamento dos descritores, em inglês: "sepsis", "hemodynamic", "corticosteroids" em português: "sepse", "hemodinâmica", "corticosteroides" e em espanhol: "sepsis", "hemodinámico", "corticosteroides", nas seguintes bases de dados: National Library of Medicine (PubMed MEDLINE), Scientific Electronic Library Online (Scielo), Cochrane Database of Systematic Reviews (CDSR), Google Scholar, Biblioteca Virtual em Saúde (BVS) e EBSCO Information Services. Totalizaram-se 24 artigos científicos para a revisão integrativa da literatura, publicados entre 2011 e 2021. Resultados a melhora na sobrevida com corticosteroides pode resultar da redução da duração do choque, gravidade da inflamação e número de disfunções orgânicas. Os benefícios demonstrados estão de acordo com os achados de estudos em animais, músculos lisos vasculares isolados e células inflamatórias e em voluntários saudáveis desafiados com endotoxina. A hidrocortisona possui vários benefícios em relação aos recursos de saúde, tais como: é uma droga barata e segura, possibilita menor permanência na UTI, menor tempo de ventilação mecânica e resolução mais rápida da sepse. Conclusão: os glicocorticoides, em especial a hidrocortisona, podem ser utilizados para o tratamento dos pacientes com sepse. Conforme as literaturas utilizadas, os resultados são satisfatórios e possuem um custeio de fácil adesão.
\end{abstract}

Palavras-chave: Corticosteroides; Sepse; Terapia intensiva.

\section{Abstract}

Introduction: sepsis is a systemic inflammatory disease associated with an infection with severe manifestations that occurs throughout the body. However, this infection does not necessarily have to be everywhere in the body, it may be restricted to just one location, but it may cause an inflammatory response throughout the body to try to control the cause of the infection. corticosteroids in the treatment of sepsis in patients hospitalized in intensive care units (ICU's). 
Methodology: this is a descriptive research of the integrative literature review type. The descriptors were crossed in English: "sepsis", "hemodynamic", "corticosteroids" in Portuguese: "sepsis", "hemodynamics", "corticosteroids" and in Spanish: "sepsis", "hemodynamic", "corticosteroids", in the following databases: National Library of Medicine (PubMed MEDLINE), Scientific Electronic Library Online (Scielo), Cochrane Database of Systematic Reviews (CDSR), Google Scholar, Virtual Health Library (VHL) and EBSCO Information Services. A total of 24 scientific articles were published for the integrative literature review, published between 2011 and 2021. Results The improvement in survival with corticosteroids may result from the reduction in the duration of shock, severity of inflammation and number of organ dysfunctions. The demonstrated benefits are in agreement with the findings of studies in animals, isolated vascular smooth muscle and inflammatory cells and in healthy volunteers challenged with endotoxin. Hydrocortisone has several benefits in relation to health resources, such as: it is a cheap and safe drug, allows for a shorter stay in the ICU, shorter mechanical ventilation time and faster sepsis resolution. Conclusion: glucocorticoids, especially hydrocortisone, can be used to treat patients with sepsis. According to the literature used, the results are satisfactory and have a costing that is easy to adhere to.

Keywords: Corticosteroids; Sepsis; Intensive therapy.

\section{Resumen}

Introducción: la sepsis es una enfermedad inflamatoria sistémica asociada a una infección de manifestaciones graves que se presenta en todo el organismo. Sin embargo, esta infección no tiene que estar necesariamente en todas partes del cuerpo, puede estar restringida a un solo lugar, pero puede causar una respuesta inflamatoria en todo el cuerpo para tratar de controlar la causa de la infección. Corticosteroides en el tratamiento de la sepsis en pacientes hospitalizados en unidades de cuidados intensivos (UCI). Metodología: se trata de una investigación descriptiva del tipo revisión integradora de la literatura. Los descriptores fueron cruzados, en inglés: "sepsis", "hemodinámica", "corticosteroides", en portugués: "sepsis", "hemodinámica", "corticosteroides" y en español: "sepsis", "hemodinámica", "corticosteroides", en las siguientes bases de datos: National Library of Medicine (PubMed MEDLINE), Scientific Electronic Library Online (Scielo), Cochrane Database of Systematic Reviews (CDSR), Google Scholar, Virtual Health Library (VHL) y EBSCO Information Services. Se publicaron un total de 24 artículos científicos para la revisión integradora de la literatura, publicados entre 2011 y 2021. Resultados La mejora de la supervivencia con corticosteroides puede deberse a la reducción de la duración del shock, la gravedad de la inflamación y el número de disfunciones orgánicas. Los beneficios demostrados están de acuerdo con los hallazgos de estudios en animales, células inflamatorias y de músculo liso vascular aislado y en voluntarios sanos desafiados con endotoxina. La hidrocortisona tiene varios beneficios en relación a los recursos sanitarios, tales como: es un fármaco barato y seguro, permite una estancia más corta en la UCI, un tiempo de ventilación mecánica más corto y una resolución de la sepsis más rápida. Conclusión: los glucocorticoides, especialmente la hidrocortisona, se pueden utilizar para tratar pacientes con sepsis. Según la literatura utilizada, los resultados son satisfactorios y tienen un costeo fácil de cumplir.

Palabras clave: Corticosteroides; Septicemia; Terapia intensiva.

\section{Introdução}

A sepse consiste em uma doença inflamatória sistêmica associada a uma infecção com manifestações graves que ocorre por todo o organismo. No entanto, essa infecção não precisa estar necessariamente em todos os locais do organismo, pode estar restrita em apenas um local, mas causar uma resposta inflamatória por todo o corpo para tentar controlar a causa da infecção. Segundo a Society of Critical Care of Medicine a resposta inflamatória sistêmica (SIRS) deve incluir alguns critérios, os quais são: febre ou hipotermia; frequência cardíaca maior que 90 bpm; taquipneia ou $\mathrm{PaCO}_{2}<32 \mathrm{mmHg}$, ou leucocitose (> 12.000), leucopenia (<4.000) ou presença de formas imaturas no leucograma (>10\%) (de Mello et al., 2011).

Nesse sentido, é importante ressaltar que sepse e choque séptico são condições diferentes. Na sepse pode ocorrer o comprometimento de vários órgãos do paciente, causando falência múltipla destes. Além disso, no quadro da sepse os macrófagos são estimulados por meio de várias vias de ativação da inflamação, sendo esse um dos motivos pelo qual o tratamento com corticosteroides é administrado nesse quadro. O choque séptico é uma forma mais grave da sepse, a inflamação alcança um estágio avançado e não possui respostas quanto ao tratamento de fluidos (Neta et al., 2020). Quanto a epidemiologia, a sepse é uma condição extremamente frequente e com custos muito elevados, sendo uma das principais causas não só de óbitos, mas também de hospitalização nas unidades de terapia intensiva (UTI's). Estima-se que a sepse tenha uma incidência mundial de 31,5 milhões de casos de sepse por ano, com cerca de 5,3 milhões de óbitos. De acordo com o estudo realizado por Taniguchi et al. (2018), nas UTI's brasileiras, algumas unidades não possuem suporte adequado para o 
tratamento de pacientes sépticos ou até mesmo alguns procedimentos básicos para monitoramento são escassos, o que contribui para uma maior taxa de mortalidade (Souza et al., 2017).

Diante disso, os corticosteroides conhecidos como agentes anti-inflamatórios e imunossupressores, são utilizados no tratamento da sepse com o objetivo de atenuar e modificar a resposta inflamatória. Esses medicamentos exercem os seus efeitos por meio da modulação da transcrição de genes inflamatórios e da imunossupressão. As células nucleadas expressam alguns receptores intracelulares de corticoides, estes, por sua vez, ligam-se aos corticoides formando um complexo esteroidereceptor que ao penetrar no núcleo modula a transcrição de certos genes. (Neta et al., 2020). Além disso, este medicamento atua inibindo o fator nucelar kappa beta $(\mathrm{NFkB})$ e a síntese de citocinas inflamatórias como o fator de necrose tumoral alfa (TNF- $\alpha$ ), interleucina 1 (IL-1) e interleucina 6 (IL-6). Outra ação dos corticosteroides consiste na diminuição da migração de células inflamatórias para os locais de lesão ou inflamação, o que reduz a expressão de moléculas de adesão endotelial e de adesão intracelular, de prostaglandinas e de quimiocinas. Sendo assim, o uso de corticosteroides para o tratamento da sepse é importante, pois a resposta inflamatória do paciente é atenuada (Ramanan et al., 2019). Sendo assim, o objetivo deste estudo é demonstrar as atuais evidências sobre o uso de corticosteroides no tratamento da sepse em paciente hospitalizados nas unidades de terapia intensiva (UTI's).

\section{Metodologia}

Trata-se de uma pesquisa descritiva do tipo revisão integrativa da literatura. Para a elaboração da questão de pesquisa da revisão integrativa utilizou a estratégia PICOT (Acrômio para Patient, Intervention, Comparation, Outcome and Time). O uso dessa estratégia para formular a questão de pesquisa na condução de métodos de revisão possibilita a identificação de palavras-chave, as quais auxiliam na localização de estudos primários relevantes nas bases de dados. Assim, a questão de pesquisa delimitada foi: "quais as evidências sobre o uso de corticosteroides no tratamento de sepse?". Dessa maneira, compreende-se que P= Indivíduos sob sepse; I = Indivíduos que utilizaram corticosteroides como intervenção para a sepse; Co = Indivíduos em tratamento para sepse sem utilização de corticosteroides; $\mathrm{T}=$ Duas semanas de coleta de dados. A partir do estabelecimento das palavras-chave da pesquisa, foi realizado o cruzamento dos descritores, em inglês: "sepsis", "hemodynamic", "corticosteroids" em português: "sepse", "hemodinâmica", "corticosteroides" e em espanhol: "sepsis", "hemodinámico", "corticosteroides", nas seguintes bases de dados: National Library of Medicine (PubMed MEDLINE), Scientific Electronic Library Online (Scielo), Cochrane Database of Systematic Reviews (CDSR), Google Scholar, Biblioteca Virtual em Saúde (BVS) e EBSCO Information Services.

A pesquisa bibliográfica foi de cunho exploratório, partindo da identificação, da seleção e da avaliação de trabalhos e de artigos científicos considerados relevantes para dar suporte teórico para a classificação, a descrição e a análise dos resultados. A busca foi realizada nos meses de setembro e outubro de 2021. Foram considerados estudos publicados no período compreendido entre 2011 e 2021. A estratégia de seleção dos artigos seguiu as seguintes etapas: busca nas bases de dados selecionadas; leitura dos títulos de todos os artigos encontrados e exclusão daqueles que não abordavam o assunto; leitura crítica dos resumos dos artigos e leitura na íntegra dos artigos selecionados nas etapas anteriores.

Foram analisadas fontes relevantes inerentes ao tema, utilizando como um dos principais critérios a escolha de artigos atuais, originais e internacionais. Após leitura criteriosa das publicações, 3 artigos não foram utilizados devido aos critérios de exclusão. Assim, totalizaram-se 24 artigos científicos para a revisão integrativa da literatura, com os descritores apresentados acima. Após esta seleção, filtraram-se por artigos dos últimos dez anos e por artigos em línguas portuguesa, espanhola e inglesa. Como critérios de inclusão, foram considerados artigos originais, que abordassem o tema pesquisado e permitissem acesso integral ao conteúdo do estudo, no período de 2011 a 2021, em inglês, espanhol e português. O critério de exclusão foi 
imposto naqueles trabalhos que não estavam em espanhol, inglês ou português, que não tinham passado por processo de PeerView e que não relacionasse a utilização de corticosteroides para o tratamento de choque séptico.

\section{Resultados e Discussão}

A sepse é caracterizada como uma resposta inflamatória sistêmica mediada por citocinas e é responsável por altas taxas de morbimortalidade, e o uso dos corticosteroides como terapia medicamentosa pode ser adequado para o manejo da sepse, pois bloqueia processos para a síntese e a circulação de mediadores inflamatórios (de Mello et al., 2011). Os glicocorticoides apresentaram potencial ação anti-inflamatória, além de estarem relacionados a melhorias no sistema cardiovascular, com potencial reversão hemodinâmica, além de estabilizarem o paciente com sepse grave (Neta et al., 2020; Souza et al., 2017). Outrossim, os glicocorticoides atenuam a resposta pró-inflamatória, limitam a resposta anti-inflamatória e preservam a imunidade inata, sendo eficaz a sua administração como uma terapia aditiva em pacientes com sepse e choque séptico (Yao et al., 2019).

Além disso, os corticosteroides induzem a retenção de sódio por meio dos receptores mineralocorticóides e glicocorticóides. Assim, são capazes de contribuir para a correção da hipovolemia, que caracteriza a fase inicial da sepse (Fang et al., 2019). Ademais, ao favorecer o acúmulo de sódio e água na parede dos vasos sanguíneos, os corticosteroides contribuirão para aumentar a resistência vascular sistêmica, restaurando, dentro de minutos a horas, por meio de vasos de efeitos não genômicos a sensibilidade ao alfa agonista com subsequente, aumento na pressão arterial média e resistência vascular sistêmica, bem como podem interferir na ativação do canal de K + dependente de ATP (Salluh et al., 2017). Assim, a capacidade de resposta aumentada às catecolaminas é mantida ao longo dos dias por meio da transrepressão de corticosteroides de genes que codificam para ciclooxigenase II (Herming et al., 2020; Lamontagne et al., 2018).

Sob essa perspectiva, nota-se que a interrupção prematura desses medicamentos pode causar rebote na inflamação e piora do estado hemodinâmico do paciente sob sepse. Ademais, sugere-se que exercem pouco ou nenhum efeito sobre a circulação pulmonar ou índice cardíaco (Ellender et al., 2020). Outrossim, os glicocorticoides também podem prevenir a falência de órgãos e reduzir a intensidade e o número de disfunções orgânicas, reduzindo a inflamação dos tecidos e desencadeando a reparação dos tecidos e melhorando a perfusão dos tecidos, podendo inibir, em pacientes sob sepse, a liberação do fator de necrose tumoral dos tecidos vasculares e músculos lisos, como também melhoram a distribuição renal de oxigênio e permeabilidade do endotélio glomerular, normalizando, assim, a depuração de água livre e excreção renal de sódio (Fuji et al., 2020; Rygård et al., 2018).

O tratamento com doses moderadas de hidrocortisona aumentou a densidade capilar e a perfusão em pacientes em choque séptico, efeitos que ocorreram dentro de uma hora após sua administração e que podem provavelmente ser resultados da suprarregulação da isoforma endotelial da sintase do óxido nítrico, sendo recomendada pelo Surviving Sepsis Campaign, uma droga relativamente barata e segura (Lian, 2020; Montes et al., 2017)). A administração pode reduzir o período de permanência dos pacientes na UTI, além de um menor período na ventilação mecânica e uma resolução rápida do choque (Moskowitz et al., 2020; Shi et al., 2020). Entretanto, o uso da hidrocortisona ainda é controverso, uma vez que existem dúvidas sobre a sua dose, duração e método de aplicação (Vandewalle et al., 2020; yao et al., 2019). Assim, perante os resultados analisados, em sua grande parte, evidenciaram benefícios no uso dos corticosteroides em pacientes com sepse, apesar da presença de efeitos colaterais pouco relevantes, como hiperglicemia, hipertensão e hipernatremia, que são de fácil controle na UTI (Taniguchi et al., 2019; Tavaré et al., 2017). Ademais, o uso de corticoides em crianças ainda não está bem estabelecido, uma vez que os estudos nesta população apresentam baixa qualidade metodológica (Lobo et al., 2019).

O papel do uso dos corticosteroides na redução da mortalidade em pacientes com sepse ainda permanece pouco esclarecido e, somado a questões abordadas como seus efeitos adversos e formas adequadas de administração ainda contribuem 
com o debate acerca do assunto (Ramanan et al., 2019). A melhora na sobrevida com corticosteroides pode resultar da redução da duração do choque (conforme mostrado pelas maiores proporções de reversão do choque nos dias 7 e 28), gravidade da inflamação e número de disfunções orgânicas (Lu et al., 2020). Os benefícios demonstrados estão de acordo com os achados de estudos em animais, músculos lisos vasculares isolados e células inflamatórias e em voluntários saudáveis desafiados com endotoxina (Rocha et al., 2011). De fato, estudos mostraram consistentemente que os corticosteroides melhoraram a contratilidade dos vasos e a hemodinâmica; evitou o recrutamento, proliferação e liberação de mediadores pró-inflamatórios por células inflamatórias; e melhora a sobrevivência de todos os tipos de modelos animais de sepse (Tagami et al., 2014). Finalmente, não evidenciou-se aumento das taxas de sangramento gastroduodenal, superinfecções ou hiperglicemia associadas ao uso de corticosteroides.

\section{Conclusão}

Cursos curtos de corticosteroides em altas doses não afetam a mortalidade por sepse grave e choque séptico e ciclos longos de corticosteroides em baixas doses melhoram a hemodinâmica sistêmica e reduzem o tempo de tratamento com vasopressores. Ademais, ciclos longos de corticosteroides em baixas doses não alteram significativamente o risco de sangramento gastroduodenal, superinfecções ou hiperglicemia, bem como atenua a resposta pró-inflamatória, limita a resposta anti-inflamatória e preserva a imunidade inata. Além disso, os glicocorticoides exógenos estão amplamente disponíveis, possuem baixo custo e capacidade para inibir a cascata inflamatória. Neste sentido, conclui-se que os glicocorticoides, em especial a hidrocortisona, podem ser utilizados para o tratamento dos pacientes com sepse. Conforme as literaturas utilizadas, os resultados são satisfatórios e possuem um custeio de fácil adesão. Contudo, o uso destes medicamentos, mesmo apresentando benefícios, ainda é controverso, devido aos efeitos colaterais causados, à posologia e à forma de administração. Desta forma, discussões acerca deste assunto ainda existem.

\section{Referências}

de Mello, A. C. P. M., et al. (2011). Corticoterapia no choque séptico e sepse grave. Revista da Sociedade Brasileira de Terapia Intensiva, 9 (1), $50-53$.

Ellender, T., et al. (2020). Updates in Sepsis Resuscitation. Emergency Medicine Clinics of North America, 38 (4), $807-818$.

Fang, D., et al. (2019). Association of corticosteroid treatment with outcomes in adult patients with sepsis: a systematic review and meta-analysis. JAMA Internal Medicine, 179 (2), 213-223.

Fuji, T., et al. (2020). Metabolic support in sepsis: corticosteroids and vitamins: the why, the when, the how. Curr. Opin. Crit. Care, 26(4), 363-368.

Heming, N., et al. (2020). Sepsis in the critically ill patient: current and emerging management strategies. Expert Review of Anti-Infective Therapy, 7 (8), 111.

Lamontagne, F., et al. (2018). Corticosteroid therapy for sepsis: a clinical practice guideline. BMJ, 9 (7), 1-14.

Lian, X. J., et al. (2019). Reevaluating the Role of Corticosteroids in Septic Shock: An Updated Meta-Analysis of Randomized Controlled Trials. Biomed Res Int., 1 (8), 56-70.

Lin, H. Y. (2020). The severe COVID-19: a sepsis induced by viral infection? And its immunomodulatory therapy. Chinese Journal of Traumatology, 9 (6), $39-45$.

Lu, X., et al. (2020). Efficacy and safety of corticosteroids for septic shock in immunocompromised patients: A cohort study from MIMIC. The American Journal of Emergency Medicine, 5 (8), 1-9.

Lobo, S. M., et al. (2019). Mortalidade por sepse no Brasil em um cenário real: o projeto UTIs brasileiras. Revista Brasileira de Terapia Intensiva, 4 (1), 3444.

Montes, P. S., et al. (2017). Uso de corticosteroides em pacientes com sepse: uma revisão bibliográfica. Revista Brasileira de Terapia Intensiva, 4 (8), 1-8.

Moskowitz, A., et al. (2020). Effect of Ascorbic Acid, Corticosteroids, and Thiamine on Organ Injury in Septic Shock: The ACTS Randomized Clinical Trial. JAMA, $324(7), 642-650$.

Neta, A. B. C. S., et al. (2020). Relações da corticoterapia no tratamento do choque séptico. Brazilian Journal of Health Review, 3 (1), $1324-1330$. 
Research, Society and Development, v. 10, n. 14, e502101422468, 2021

(CC BY 4.0) | ISSN 2525-3409 | DOI: http://dx.doi.org/10.33448/rsd-v10i14.22468

Ramanan, M., et al. (2019). Steroids and Sepsis: The Debate Continues. International Anesthesiology Clinics, 57 (2), $17-30$.

Rocha, S. L., et al. (2011). Resposta hemodinâmica após uso de corticoide na sepse experimental. Revista do Médico Residente, 13 (1), 1-11.

Rygård, S. L., et al. (2018). Low-dose corticosteroids for adult patients with septic shock: a systematic review with meta-analysis and trial sequential analysis. Intensive Care Med, 44 (7), 1003-1016.

Salluh, J., et al. (2017). Corticosteroids in Severe Sepsis and Septic Shock. Shock, 47 (1), 47-51.

Shi, R., et al. (2020). Benefit of hydrocortisone, thiamine, and vitamin C for patients with sepsis or septic shock? Too early to draw conclusions. Critical Care, 24(1), 18-28.

Souza, C. N. S., et al. (2017). Sepse em ambiente hospitalar. Brazilian Journal of Health Review, 1 (7), 15-24.

Tagami, T., et al. (2014). Low-dose corticosteroid use and mortality in severe communityacquired pneumonia patients The European Respiratory Journal, 45 (2), 463-472.

Taniguchi, L. U., et al. (2019). Disponibilidade de recursos para tratamento da sepse no Brasil: uma amostra aleatória de instituições brasileiras. Revista Brasileira de Terapia Intensiva, 31 (2), 193-201.

Tavaré, A., et al. (2017). Recognition, diagnosis, and early management of sepsis: NICE guideline. Br J Gen Pract, 67 (1), $185-166$.

Vandewalle, J., et al. (2020). Glucocorticoids in Sepsis: To Be or Not to Be. Front. Immunol., 11 (13), 1-11.

Yao, Y., et al. (2019). Are corticosteroids beneficial for sepsis and septic shock? based on pooling analysis of 16 studies. Frontiers in pharmacology, 10 (8), $714-$ 